Original Article

\title{
THE CLINICAL EFFICACY OF AUTOLOGOUS CONCENTRATED PLATELETS IN TREATM ENT OF TMJ DISORDERS - A PILOT STUDY
}

\author{
S.M. Sharma ${ }^{1} \&$ Dhruvit Thakar ${ }^{2}$ \\ ${ }^{1}$ Head of the Department, ${ }^{2}$ Junior Resident, Department of Oral and M axillofacial Surgery \\ A.B. Shetty M emorial Institute of Dental Sciences, Nitte University, M angalore - 575018 \\ Karnataka, India. \\ Correspondence : \\ Dhruvit Thakar \\ Junior Resident, Department of Oral and M axillofacial Surgery \\ A.B. Shetty M emorial Institute of Dental Sciences, Nitte University, M angalore - 575 018, Karnataka, India. \\ Mobile : +9197425 59615 E-mail : drdhruvitthakar@gmail.com
}

\begin{abstract}
:
Platelet rich plasma is a natural concentrate of autologous blood growth factor which is obtained by sequestering and concentrating platelets by gradient density centrifugation experimented in different fields of medicine in order to test its potential to enhance tissue regeneration. These platelets when activated undergo degranulation to release growth factors with healing properties. It also contains plasma, cytokines, thrombin, and other growth factors that are implicated in wound healing and have inherent biological and adhesive properties. The aim of the study is to explore a novel approach to treat degenerative lesion of the articular cartilage of temporomandibular joint. Patients with chronic degenerative conditions of the joint, were treated with intra articular PRP injections. The procedure involved collection of $10 \mathrm{ml}$ of venous blood and twice centrifuged to obtain $2 \mathrm{ml}$ of PRP which was used for injection after activation by calcium chloride. The patients were clinically prospectively evaluated before and at the end of the treatment, and at 2,4 , and 6 month of follow up. Inter incisical mouth opening, clicking of temporomandibular joint and VASfor pain were used for clinical evaluation. The results showed significant improvement of all clinical scores from basal evaluation to the end of the therapy and on follow up at 2-6 months. The preliminary results indicate that the treatment with PRP injections are safe and has the potential to reduce pain and improvement joint function with low degree of articular degeneration.
\end{abstract}

Keywords : PRP-Articular Cartilage-Temporomandibular Joint-Intra Articular Injections.

\section{Introduction:}

The incidence of articular cartilage damage has grown due to greater physical activity in all age group ${ }^{1}$. Unfortunately articular cartilage lesions, with their limited inherent healing potential are hard to treat ${ }^{2,3,4,5}$.

A variety of agents, such as non-steroidal antiinflammatory drugs, chondroitin sulphate, hyaluronic acid and glucocorticoids have been proposed as an noninvasive solution for pain treatment, improvement in

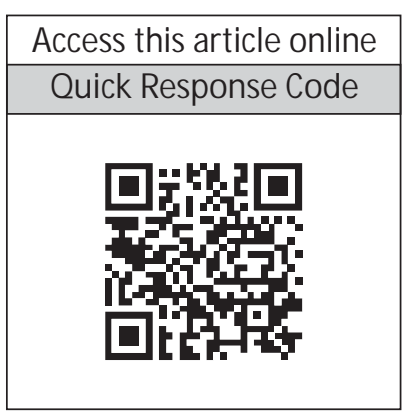

function and disability and ultimate modification ${ }^{6}$ of severe chondral degeneration and osteoarthritis with varying success rates.

Current research is investigating new methods for stimulating repair or replacing damaged cartilage, such as matrix metalloproteinase inhibitor, gene therapy, cytokine inhibitor, artificial cartilage substitute and growth factors.

Autologous Concentrated Platelets is a natural concentrate of growth factors from blood. It is observed that these preparations had bone forming properties as well as antiinflammatory and antibacterial properties ${ }^{13,14}$.

Platelets aside from their role in coagulation and hemostasis, they contain $\alpha$-granules with various molecules which are secreted upon their activation. These molecules are growth factors (Platelet derived growth factor, transforming growth factor $ß$, vascular endothelial growth factor), endostatins, platelet factor 4, angiopoietins and thrombosponding 1. All of these are involved in healing process. 
M oreover platelets have been identified to have analgesic properties by releasing protease activated receptor 4 peptides $^{16}$.

The aim of the pilot study was to evaluate the safety of our protocol, by assessing the number, timing, severity, duration and resolution of adverse effects.

The second aim of this study was to analyse the short term results obtained, to determine feasibility, indication criteria, and application modalities for further wider studies.

The study was done to evaluate efficacy of autologous concentrated platelets in treatment of degenerative disease of temporomandibular joint with respect to pain, mouth opening and mastication.

\section{M aterials and Methodology:}

The first step of the procedure involves withdrawal of $10 \mathrm{ml}$ of patient venous blood in a vaccutainer containing sodium citrate.

Platelet count is also done at the time of initial blood draw to ensure the counts are within normal limits.

Patients with any platelet disorder, who have taken NSAIDS in past one week, or who have undergone corticosteroid injection intra-articular in last two months were not included in the study.

After blood withdrawal it is transferred into centrifuge tube under sterile aseptic condition.

After which PRP (Platelet Rich Plasma) is prepared from whole blood by double centrifugation cycle. ${ }^{17}$

The first cycle involves centrifugation of whole blood at 1800 rpm for 15 minutes to separate erythrocytes.

After centrifugation, the blood is separated into 3 layers, the lowermost is red layer containing erythrocytes, and middle layer is white containing leucocytes and inflammatory cytokines. The topmost layer is yellow made up of plasma, platelets and growth factors.
The topmost layer is transferred into another centrifuge tube, while the lower two layers are discarded.

Now the topmost layer in another tube is centrifuged at 3500 rpm for 10 minutes to concentrate platelets.

At the end of the procedure $2 \mathrm{ml}$ of concentrated PRP is obtained.

Now before injecting PRP, $10 \%$ of $\mathrm{CaCl}_{2} \mathrm{C}^{2+}=$ $0.22 \mathrm{mEq} \times$ dose) is added to PRP unit for activation of the platelets.

\section{Treatment Procedure and Follow Up}

The prepared platelet concentrate was injected into the upper joint space according to Mccains protocol of performing arthroscopy, but only the posterior point was used to enter the joint space and pump PRP. ${ }^{18}$

A line is drawn from middle of the tragus to the outer canthus. The posterior entrance point is located along the canthotragal line, $10 \mathrm{~mm}$ from the middle of the tragus and $2 \mathrm{~mm}$ below the line.

First the zygomatic arch is palpated and the mandibular condyle is forced into anterior position by the assistant and the preauricular concavity is formed at the marking point of the injection.

Following these $2 \mathrm{ml}$ of PRP is injected into the superior joint space, with the needle being aimed medially and slightly anterio superiorly until the contact with glenoid fossa is achieved.

After this PRP is injected into the joint space and the needle is withdrawn.

At the end of the procedure, the patient was asked to open and close the mouth several time for a minute to ensure equal distribution of PRP before it converts into gel.

After the injection the patient was sent home with the advice to continue on oral soft diet for next 3 days and gradually resume to normal diet after 3 days.

All the post-operative complications and adverse effects where recorded. 
These patients where recalled for follow up at 2, 4 and 6 months after the procedure was carried out.

\section{Results:}

No major adverse events related to injections were observed during treatment and follow-up period. Patients presented with mild pain and swelling at the site of the injection for one week after injection which resolved by itself within a period of week without any intervention. There were no sign of infection or restriction of the joint movement.

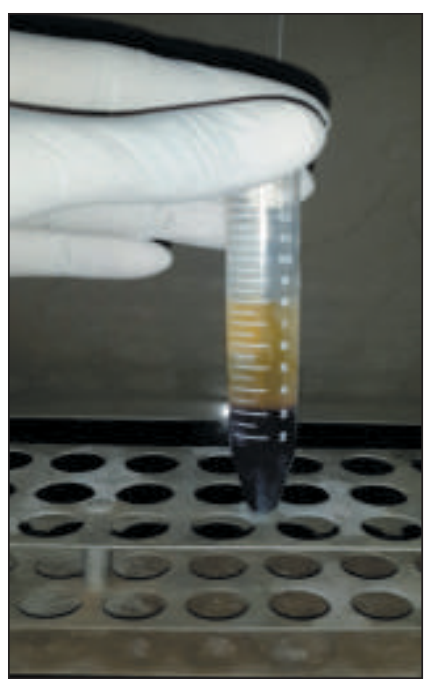

Figure 1 : First Centrifuge

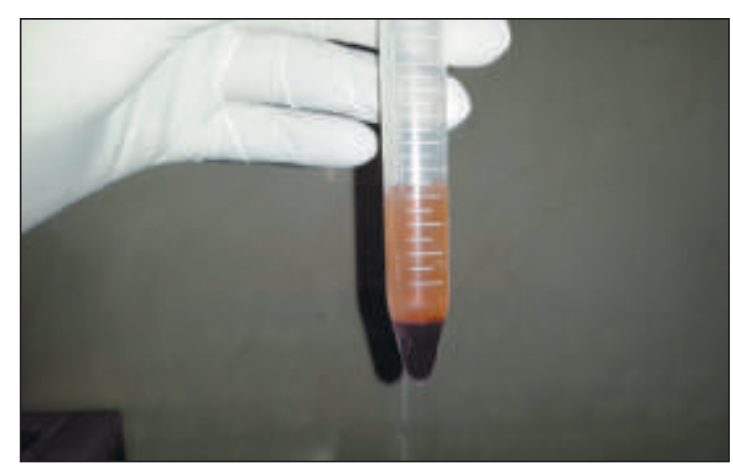

Figure 2 : Second Centrifuge

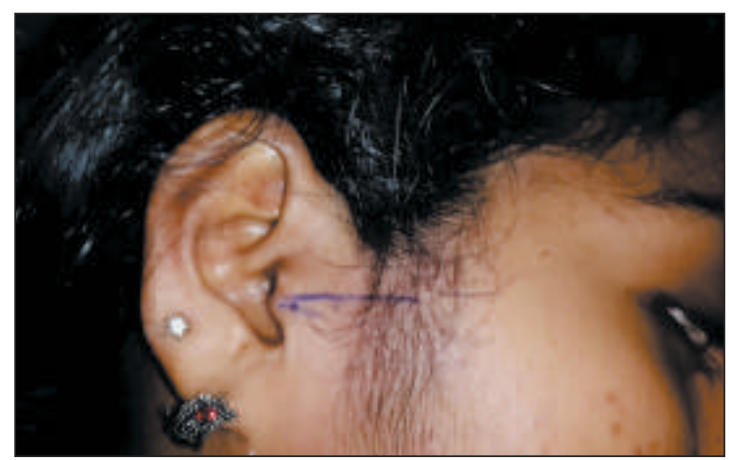

Figure 3 : M arkings according to McCains Protocol

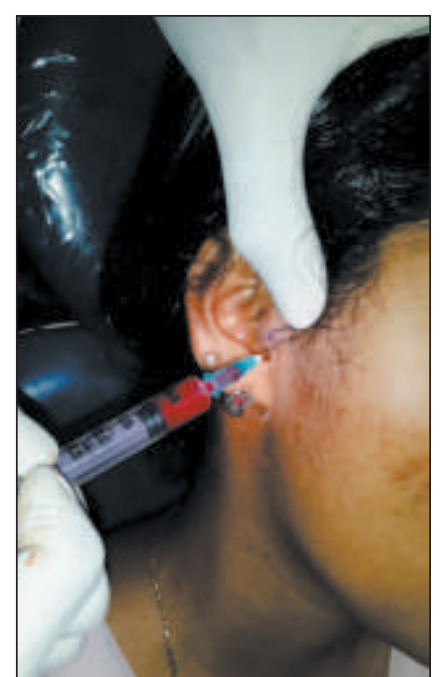

Figure 4 : PRP Injection in upper joint Space of TM J

\section{Discussion:}

The most important finding of the present study was to investigate this novel biological approach for treatment of degenerative temporomandibular joint. In recent years, there has been an increasing prevalence of use of autologous blood products that might provide cellular and humoral mediators to favour tissue healing in a variety of applications. The rationale is based on the activity of blood growth factors. The growth factors are diverse group of polypeptides that have important role in regulation of growth and tissue development, determination of behaviour of cells, including chondrocytes. The understanding of their effects on chondrocytes is rapidly progressing and many growth factors have been identified as aiding in regulation of articular cartilage. Most of the studies include the transforming growth factor-beta super family (TGF-ß), platelet-derived growth factor (PDGF), insulin like growth factor (IGF), fibroblast growth factor (FGF), and hepatocyte growth factor (HGF). ${ }^{19}$

In particular, TGF- $ß$ is one of the most important factor involved in the process of cartilage regeneration; its function includes increased chondrocyte phenotype 
expression. ${ }^{(20,21)}$, the chondrocyte differentiation of mesenchymal stem cells ${ }^{(7,22)}$, matrix deposition ${ }^{8}$ and counteract with most of the suppressive effects of inflammatory mediators IL 1 on cartilage specific macromolecules synthesis ${ }^{20}$. PDGF also plays an important role in the maintenance of hyaline like chondrogenic phenotype, increases chondrocyte proliferation, upregulation of proteoglycan synthesis, and is a potent chemotactic factor for all cells of mesenchymal origin ${ }^{23}$. IGF is another important cartilage anabolic factor ${ }^{24}$ and it has an important role in augmenting the effects of other growth factors found in cartilage ${ }^{7,19}$. M any other growth factors are involved in cartilage regeneration and metabolism, like FDG and HGF, and they may have chondroinductive actions, independently or more so with additive effects and synergistic interaction ${ }^{19}$. PRP is a blood product that allows in a simple, low cost, and minimally invasive way to obtain a concentration of many of these growth factors ${ }^{18,9,10,}$ ${ }^{11,}{ }^{12}$. Platelets contain storage pools of growth factors including PGDF, TGF $B$, IGF-1, FGF and many others. Cytokines, chemokines, and newly synthesised metabolites are also released ${ }^{25}$. PRP derived from centrifugation of blood have platelet concentration four to five time higher than normal blood concentration. The platelet concentrate is activated by addition of calcium chloride, and this results in formation of platelet gel provides an adhesive support that can confine secretion to a chosen site ${ }^{15}$.

Blood derived growth factors have already been studied for their potential in helping cartilage repair and documented in literature. Frisbie ${ }^{26}$ administered autologous concentrated in horses with experimentally induced osteoarthritis, obtaining significant clinical improvement in lameness, decreased synovial membrane hyperplasia, less gross cartilage fibrillation, and synovial membrane hemorrhage and increased synovial fluid concentration of interleukin 1 receptor antagonist. Graissamaier ${ }^{27}$ investigated the effect of human platelet supernatant on chondrocyte proliferation and differentiation and concluded the addition may accelerate chondrocyte expansion, even though it can also lead to their dedifferentiation. Saito ${ }^{28}$ documented preventive effects against osteoarthritis progression with administration of gelatine hydrogel microspheres containing PRP in rabbit model. PRP has also been used as an injectable scaffold for tissue engineering.

These studies and others suggest an important role these biological regulators of chondrocyte on cartilage repair. However for the time being, the evidence base for the use of PRP is in infancy and there are very few paper that address these treatment.

The primary objective of our study was to evaluate the safety of our protocol, by gathering and assessing the number, timing, severity, duration and resolution of the related adverse effects.

No complication such as infection, marked muscle atrophy, fever, hematoma, tissue hypertrophy, adhesion formation or other major events occurred in the subjects. Only minor complication like pain and swelling at the site of injection which was present for a period of week and resolved by itself without any active intervention.

The second aim of the study was to evaluate the effectiveness of PRP in treatment of TMJ disorders with respect to pain, mastication, clicking and interincisal mouth opening. Results showed significant improvement in all the score and the results obtained remained stable over a period of 2-6 months of follow up. Promising results were obtained about safety, feasibility, and short term effectiveness of the treatment protocol.

These reported document of our experience on use of autologous platelet rich plasma concentrate- derived growth factor injections in TMJ as a treatment of cartilage degeneration, represent a low invasive and safe alternative in patient with early stage osteoarthritis. The hypothesis behind the treatment is that PRP injections reduces the inflammatory and degenerative articular processes of the joint, leading to improved function of the joint.

\section{Conclusion:}

The preliminary short term results of our pilot study are 
encouraging and indicate the treatment with autologous PRP intra articular injection is safe and is useful for treatment of early degenerative articular pathology of TMJ, aiming to reduce pain and improving function and quality of life. However further randomised controlled studies are required to confirm the real potential and to

\section{References:}

1. Curl WW, Krome J, Gordon ES, Rushing J, Smith BP, Poeling GG (1997) Cartilage injury: a review of 31,516 knee arthroscopies. Arthroscopies 13:456-460

2. Alford JW, Cole BJ (2005) Cartilage restoration, part I: basic science, historical perspective, patient evaluation, and treatment options. Am J Sports Med 33:295-306

3. Buckwalter JA, Brown TD (2004) Joint injury, repair, and remodelling: roles in post-traumatic osteoarthritis. Clin Orthop Relat Res 427:7-16

4. Ochi M, Ucho Y, Kawasaki K, Wakitani S, Iwasa J (2002) Transplantation of cartilage-like tissue made by tissue engineering in treatment of cartilage defects of knee. J Bone Joint Surg Br 84:571-578

5. Sgaglione NA, Miniaci A, Gillogly SD, Carter TR (2002) Update on advanced surgical techniques in treatment of traumatic focal articular cartilage lesion in the knee. Arthroscopy 18:9-32

6. Hayami T (2008) Osteoarthritis of knee joint as a cause of musculoskeletal ambulation disability symptom complex (M ADS). Clin Calcium 18:1574-1580

7. Ulrich-Vinther M, Maloney MD, Schwarz EM, Rosier R, O'Keefe RJ (2003) Articular cartilage biology. J Am Acad Orthop Surg 11:421-430

8. Fazer A, Bunning RA, Thavarajah M, Seid JM, Russell RG (1994) Studies on type II collegen and aggercan production in human articular chondrocytes in vitro and effects of tranforming growth factor-beta and interlukin-1beta. Osteoarthr cartil 2:235-245

9. Grimaud E, Heymann D, Redini F (2002) Recent advance in TGF-beta effect on chondrocyte metabolism.Potential therpeutic roles of TGFbeta in cartilage disorder. Cytokine Growth Factor Rev 13:241-257

10. Hickey DG, Frenkel SR, Di Cesare PE (2003) Clinical application of growth factor for articular cartilage repair. Am J Orthop 32:70-76

11. Schmidt M B, Chen EH, Lynch SE (2006) A review of effects of insulinlike growth factors and platelet derived growth factors on in vivo cartilage healing and repair. Osteoarth Cartil 14:403-412

12. Song SU, Hong YJ,Oh IS, Yi Y, Choi KB, Lee JW, Park KW, Han JU, SUJ JK, Lee KH (2004) Regenration of hyaline articular cartilage with irradiated transforming growth factor beta-1 producing fibroblast. Tissue Engg 10:665-672

13. Anitua E. Plasma rich in growth factors: Preliminary result of use in preparation of future sites of implants. Int J Oral Maxillofac Implants 1999;14:529-535.

14. Bielecki TM , Gazdzik TS, Arendt J, Szczepanski T, Krol W, Wielkoszynski T. Antibacterial effect of autologous platelet gel enriched with growth factors and other active substance: An in vitro study. J Bone Joint Surg $\operatorname{Br} 2007 ; 89: 417-420$

15. Anitua E, Andia I, Ardanza B, Nurden P, Nurden AT. Autologous platelets as a source of protein for healing and tissue regeneration. Thromb Haemost 2004;91:4-15.

16. Asfaha S, Cenac N, Houle S, et al. Protease-activated receptor-4: aA novel mechanism of inflamatory pain modulation. $\mathrm{Br} J$ Pharmacol evaluate the durability of the procedure and to better identify the indication criteria.

Also further studies are required to confirm the adequate dosage of PRP and the frequency of the injections required to achieve long term results.

2007;150:176-185.

17. Elizaveta Kon, Robert Buda, Gluseppe Filardo et al. Platelets-Rich Plasma: Intra-articular Knee Injections produced favorable results on degenerative cartilage lesion. Knee surg sports traumatol arthsoc octo 2009;1-8

18. M urakami KL, lizuka T, M atsuki M, et al. Recapturing the persistently anteriorly displaced disk by mandibular manipulation after pumping and hydraulic pressure to the upper joint cavity of temporomandibular joint. Cranio 1987;5(1):17-24

19. O' Keefe RJ, Crabb ID, Puzas JE, Rosier RN(1994). Effects of transforming growth factor-beta 1 and fibroblast growth factor on DNA synthesis in growth plate chondrocytes are enhanced by insulin like growth factor-1.J Orthop Res 12;299-310.

20. Pujol JP, Chadjichristos C, Legendre F, Bauge C, Beauchef G, Andriamanalijaona R, Galera P, Boumediene K (2008). Interleukin-1 and transforming growth factor beta- 1 as crucial factors in osseoarthritic cartilage metabolism. Connect tissue Res 49:293-297.

21. Song SU, Cha YD, Han JU, Oh IS, Choi KB, Lee JW et al(2005) Hyaline cartilage regeneration using mixed human chondrocytes and transforming growth factor beta-1 producing chondrocytes. Tissue Eng 11:1516-1526.

22. Noth U, Rackwitz L, Heymer A, Weber M, Baumann B, Steinert A et al (2007). Chondrogenic differentiation of human mesenchymal stem cells in collagen type I hydrogel. J Biomed M ater ResA 83:626-635.

23. Schmidt M B, Chen EH, Lynch SE (2006). A review of effects of insulin like growth factor and platelet derived growth factor on in vivo cartilage healing and repair. Ostoarthr Cartil 14:403-412.

24. Martin JA, Buckwalter JA (2000) The role of chondrocyte-matrix interaction in maintaining and repairing articular cartilage. Biorheology 37:129-140.

25. Sanchez M , Sheridan P, Kupp L (2003) Is platelet rich plasma a perfect enhancement factor? A current review. Int J Oral and Maxillofac Implants 18:93-103.

26. Frisbie DD, Kawcak CE, Werpy NM, Park RD, M cllwraith CW (2007) Clinical, biochemical, and histological effects of intra articular administered of autologous conditioned serumin horses with experimentally induced osteoarthritis.

27. Gaissmaier C, Fritz J, Krachhardt T, Flesch I, Aicher WK, Ashammakhi N (2005) Effect Of platelet rich supernatant on proliferation and matrix synthesis of human articular chondrocyte in monolayer and three dimensional alginate cultures. Biomaterial 26:1953-1960.

28. Saito M, Takahashi KA, Arai Y, Inoue A, Sakao K, Tonomura H, Honjo K, Nakagawa S et al. (2009) Intraarticular administration of platelet rich plasma with biodegradable gelatin hydrogel microspheres prevents osteoarthritis progression in rabbit knee. Clin Exp Rheumatol 27:201207. 